reactivation (Schlesinger, 1966) gained conformational stability in urea approaching closely that of $E$. coli wild-type enzyme.

This information suggests that the position of zinc may restrain the initiation of helix-coil transition even on transfer to a less polar, strongly hydrogen-bonding, solvent. Zinc deficiency permits randomization to a high degree, leaving less than $3 \%$ residual $\alpha$-helix content. The ability of zinc atoms to constrain the natural conformation under conditions of stress suggests that zinc atoms are bonded to residues widely separated in the primary sequence.

In the aromatic side-chain absorption region at least four weak ellipticity bands were discernible. These bands were less pronounced in the mutant, but approached those of the wild-type on partial activation with $\mathrm{Zn}^{2+}$ and phosphate.

$E$. coli $\mathrm{C}_{10} \mathrm{~F}_{18}$ was kindly given by $\mathrm{Dr}$ A. Torriani. C.N.A.T. is grateful for a Science Research Council Studentship.

Plocke, D. J., Levinthal, C. \&Vallee, B. (1962). Biochemistry, $1,373$.

Reynolds, J. A. \& Schlesinger, M. J. (1967). Biochemistry, 6, 330.

Schlesinger, M. J. (1966). J. biol. Chem. 241, 3181.

Simpson, R. T. \& Vallee, B. L. (1968). Biochemistry, 7, 4343.

\section{Insulin Gonformations}

By S. Lewrn. (Physical Biochemistry Laboratory, Waltham Forest Division, North East London Polytechnic, London E.17)

It is generally recognized that the $\mathbf{A}$ chain and the B chain of insulin $(a)$ each contain helical conformations and $(b)$ are joined by two disulphide bonds, namely CyS $(\mathrm{A}, 7)-\mathrm{CyS}(\mathrm{B}, 7)$ and $\mathrm{CyS}$ $(A, 20)-C y S(B, 19)$ (for extensive references see, e.g., Scheraga, 1961; Rieser, 1967).

An additional intrachain disulphide linkage, namely CyS (A,6)-CyS (A,11), also exists. Further, it has been suggested that some tyrosine hydroxyl groups may be hydrogen-bonded, and in particular that this could involve tyrosine $(B, 16)$ with glutamic acid $(\mathrm{B}, 13)$.

In attempting to investigate the stereochemical and thermodynamic permissibilities, we have constructed numerous correctly proportioned molecular models ['ball and spoke', Courtauld \& CoreyPauling-Koltun (Koltun, 1965) atomic models (Lewin, 1967, 1969a,b)]. From several of these it became clear that the guanidinium group of arginine $(B, 22)$ can form a strong resonating ionic linkage with asparagine $(A, 21)$ in insulin. This conformation appears to cover one side of the inter- chain disulphide bridges, namely CyS (A,20)CyS $(B, 19)$, and therefore could afford it partial protection against rupture.

It can be computed that the above guanidinium (arginine)-carboxylate linkage is likely to be ruptured on addition of other guanidinium-groupcontaining compounds such as polyguanides and protamines, or doubly positively charged cations such as $\mathrm{Zn}^{2+}$, or doubly negatively charged multioxyanions such as sulphate. As the rupture of this ionic bond involves exposure of these groups, the possibility arises that these may result in modification of the antigenic properties of insulin. It is therefore relevant to note that Moloney, Aprile \& Wilson (1964) have concluded that the antigenicity of insulin was considerably decreased on sulphation. It is also relevant to consider whether commercial preparations of insulin containing zine and protamine differ in their biological effects from those of pure insulin, not only with respect to prolonged 'activity' insofar as the treatment of diabetes is concerned, but also with respect to intrinsic antigenic properties.

Koltun, W. L. (1965). Biopolymers, 3, 665.

Lewin, S. (1967). J. theorel. Biol. 17, 181.

Lewin, S. (1969a). J. theoret. Biol, 23, 279.

Lewin, S. (1969b). Proc. 16th annu. Colloq. Protides of the Biological Fluids, p. 51. Oxford: Pergamon Press Ltd.

Moloney, P. J., Aprile, M. A. \& Wilson S. (1964). J. New Drugs, 4, 258.

Rieser, P. (1967). Insulin, Membranes and Metabolism. Baltimore: Williams and Wilkins Co.

Scheraga, H. A. (1961). Protein Structure. London: Academic Press (Inc.) Ltd.

\section{Order of Strength of Ionic Linkages in Protein Interactions}

By S. Lewin. (Physical Biochemistry Laboratory, Waltham Forest Division, North East London Poly. technic, London E.17)

Proteins are known to be involved in various ionic linkages, and evaluation of the relative order of strength of these linkages is desirable. The order can be assessed by using stereochemical and physicochemical considerations of the probability of $(a)$ the existence of the charge and $(b)$ the direction of the electric field in appropriate cases: the latter can influence enforcement of particular conformations. Two factors assist in this evaluation: (1) resonance can play an important part in restricting the direction of the electric field, e.g. in carboxylate, diesterified phosphate and guanidinium (arginine), where the most probable direction is at right-angles to the line joining the centres of the two atoms mainly involved in the charge distribution; (2) co-operative 